\title{
Evolution of InAs/GaAs QDs Size with the Growth Rate: A Numerical Investigation
}

\author{
Bouraoui Ilahi, ${ }^{1,2}$ Manel Souaf, ${ }^{2}$ Mourad Baira, ${ }^{2}$ Jawaher Alrashdi, ${ }^{1}$ Larbi Sfaxi, ${ }^{2}$ \\ Abdulaziz Alhazaa, ${ }^{1}$ and Hassen Maaref ${ }^{2}$ \\ ${ }^{1}$ King Saud University, Department of Physics \& Astronomy, College of Sciences, Riyadh 11451, Saudi Arabia \\ ${ }^{2}$ Université de Monastir, Laboratoire de Micro-Optoélectronique et Nanostructures, Faculté des Sciences, \\ Avenue de l'Environnement, 5019 Monastir, Tunisia
}

Correspondence should be addressed to Bouraoui Ilahi; bilahi@ksu.edu.sa

Received 2 June 2015; Revised 5 August 2015; Accepted 13 August 2015

Academic Editor: Paulo Cesar Morais

Copyright (c) 2015 Bouraoui Ilahi et al. This is an open access article distributed under the Creative Commons Attribution License, which permits unrestricted use, distribution, and reproduction in any medium, provided the original work is properly cited.

\begin{abstract}
This paper investigates the impact of the deposition rate on the mean buried InAs/GaAs quantum dots' (QDs) size by means of a coupled photoluminescence spectroscopy and numerical approach. The proposed method consists in tuning the theoretical transition energies by changing the QDs aspect ratio towards best fit of the photoluminescence emission energies arising from the state filling effect. The electron-hole confined states are obtained by solving the single particle one band effective mass Schrödinger equation in cylindrical coordinates for a lens shaped QD by finite element method taking into account the strain effects. The obtained evolution is in agreement with morphological data taken from similar uncapped QDs samples.
\end{abstract}

\section{Introduction}

Self-assembled semiconductor QDs have been a subject of numerous investigations owing to their promising potential application in optoelectronic devices [1-3]. The exploitation of this kind of nanostructures towards the improvement of the devices performance mainly relies on the ability to control their size and uniformity. Accordingly a lot of works have been deployed for best optimization of the QDs properties by varying growth temperature [4], growth rate [5-7], and capping conditions [8-10]. Nevertheless, the selforganization nature of the InAs/GaAs QDs makes their properties strongly dependent on the fabrication conditions [11-14]. Various techniques have been used to evaluate the morphology of nanostructures after capping, but all have their limitations [15]. For example, while it is well recognized that decreasing the growth rate enhances the dots size and decreases their surface density $[6,7]$, no clear picture has been established yet concerning the impact of this parameter on the evolution of the buried dots aspect ratio. Furthermore, intense theoretical and computational efforts have so far been expended in order to understand the evolution of the selfassembled QD's electronic structures dependence on several parameters such as shape [16], composition [17], and wetting layer thickness [18]. Most of these works were mainly focused on fundamental investigation of the QDs electronic structure. We propose in this work to extend the numerical modeling to a more practical use by coupling it to PL measurements to gain useful estimation on the buried dots average size in a facile and nondestructive way.

In this context, we report on a coupled numerical and high excitation power photoluminescence measurements method allowing an accurate assessment of the buried QDs size and study its evolution as a function of the growth rate.

\section{Theoretical Considerations}

The theoretical calculations have been performed by COMSOL Multiphysics software [19] where the single band effective mass Schrödinger equation has been solved for a lens shaped InAs QDs using the finite element methods (FEM). A schematic representation of the modeled QD structure is 


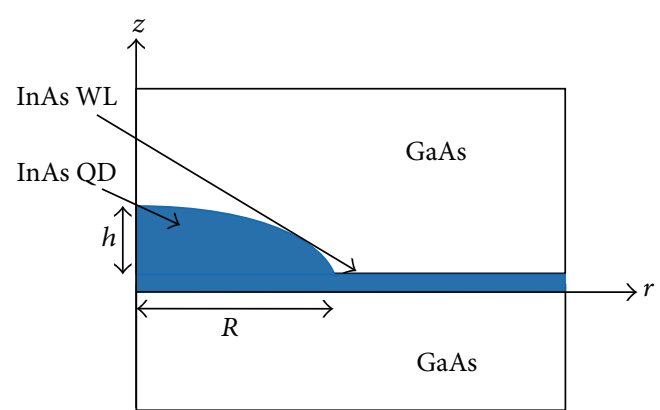

FIGURE 1: Schematic presentation of the modeled lens shaped InAs QDs with InAs wetting thickness of $1 \mathrm{ML}$.

shown by Figure 1 where the three-dimensional QD can be constituted by a rotation of $2 \pi$ around $z$-axis.

The steady state Schrödinger equation within the single band effective mass approximation can be given by

$$
\frac{-\hbar^{2}}{2 m^{*}} \Delta \psi(\vec{\rho})+V(\vec{\rho}) \psi(\vec{\rho})=E \psi(\vec{\rho}),
$$

where $\hbar, m^{*}, E, V$, and $\psi$ are, respectively, the reduced Plank's constant, the electron (hole) effective mass, confined energy state, confining potential, and wave function. $\vec{\rho}$ is the coordinate vector in spherical symmetry depending on the variables $(r, \varphi, z)$, where $r$ is the radial coordinate perpendicular to the growth direction, $z$ is the axial coordinate, and $\varphi$ is the azimuthal angle ranging from 0 to $2 \pi$.

Equation (1) can be written in 3D cylindrical coordinates as follows:

$$
\begin{aligned}
& \frac{-\hbar^{2}}{2 m^{*}}\left(\frac{\partial^{2}}{\partial r^{2}}+\frac{1}{r} \frac{\partial}{\partial r}+\frac{1}{r^{2}} \frac{\partial^{2}}{\partial \varphi^{2}}+\frac{\partial^{2}}{\partial z^{2}}\right) \psi(r, \varphi, z) \\
& +V \psi(r, \varphi, z)=E \psi(r, \varphi, z)
\end{aligned}
$$

by considering that the wave function is separable on

$$
\psi(r, \varphi, z)=\chi(r, z) \Phi(\varphi), \quad \text { where } \Phi(\varphi) \propto e^{-\mathrm{in} \varphi} .
$$

To satisfy the condition $\Phi(\varphi)=\Phi(\varphi+2 \pi), n$ must be integer.

These conditions permit, after straightforward simplification process, rewriting (2) in the following form:

$$
\begin{aligned}
& \frac{-\hbar^{2}}{2 m^{*}}\left(\frac{\partial^{2} \chi_{n}(r, z)}{\partial r^{2}}+\frac{\partial^{2} \chi_{n}(r, z)}{\partial z^{2}}\right) \chi_{n}(r, z) \\
& +\left(V+\frac{1}{r^{2}} \frac{\hbar^{2}}{2 m^{*}} n^{2}\right) \chi_{n}(r, z) \\
& \quad-\frac{\hbar^{2}-\hbar^{2}}{2 m^{*}} \frac{1}{r} \frac{\partial \chi_{n}(r, z)}{\partial r}=E_{n} \chi_{n}(r, z) .
\end{aligned}
$$

The impact of strain has been taken into account [20] in this model. The potential energy $(V)$ for electrons (holes) is taken to be zero inside the QD and equal to the strained conduction (valence) band discontinuity outside the QD. The evaluated electron and holes confined energies allow the estimation of the $n$th noncorrelated electron-hole optical transition energy by the following relation:

$$
E_{n}=e_{n}+E_{g}^{\mathrm{InAs}}+h_{n}
$$

where $E_{g}^{\mathrm{InAs}}$ is the strained InAs band gap energy. $e_{n}$ and $h_{n}$ are, respectively, the confined electrons and holes energies.

The material parameters used in this work are taken from [21].

To ensure reliable calculations, an adaptive mesh refinement has been adopted providing more grids in the active material in and around the QD and the WL. Dirichlet boundary conditions have been applied for the external boundaries where the wave function should vanish and Newman condition to the internal boundary ensuring the continuity of the wave function. More details on the calculation procedure can be found elsewhere [22].

\section{Experiments}

The studied samples consist of InAs QDs layer with 2.4 ML nominal thickness grown at $500^{\circ} \mathrm{C}$ by molecular beam epitaxy on semi-insulating (001) GaAs substrate. The samples differ on the InAs material deposition rates being varied from $0.013 \mathrm{ML} / \mathrm{s}$ to $0.069 \mathrm{ML} / \mathrm{s}$. The QDs are covered by $50 \mathrm{~nm}$ of $\mathrm{GaAs}$ at $500^{\circ} \mathrm{C}$ with a relatively high growth rate $(0.98 \mathrm{ML} / \mathrm{s})$ for all samples to minimize the In/Ga intermixing. The optical properties are evaluated by photoluminescence (PL) spectroscopy using a $514.5 \mathrm{~nm}$ line of an $\mathrm{Ar}^{+}$laser. The PL emission was dispersed by a spectrometer and detected by an InGaAs photodetector using a conventional lock-in technique. More details on the growth procedure, photoluminescence, and atomic force microscopy investigations of these structures can be found elsewhere [7].

\section{Results and Discussion}

Figure 2(a) shows typical excitation power dependent PL spectra measured at $10 \mathrm{~K}$ from the single layer QDs deposited with a growth rate of $0.069 \mathrm{ML} / \mathrm{s}$. This spectra unarguably show that the excited states get progressively populated upon increasing the excitation power density leading to the appearance of two excited states emission bands. The manifestation of multiple peaks arising from the state filling due to the three-dimensional carrier confinement allows an accurate estimation of the buried QDs size by adjusting the calculated transition energies to the experimental values. Typical envelop function for the ground states (GS) and first (1st ES) and second (2nd ES) excited states are shown in Figure 2(b).

The numerically driven emission energies deduced from (5) can be tuned by changing the QDs AR. Good matching between the theoretical and experimental emission bands within some meV yielded an estimation of the QDs average height of $3.3 \mathrm{~nm}$ and base diameter of $29 \mathrm{~nm}$.

The PL investigation of the QDs fabricated at different growth rate has also revealed a state filling effect with varying the excitation power density. Typical PL spectra showing the emission bands relative to the ground state (GS) and 


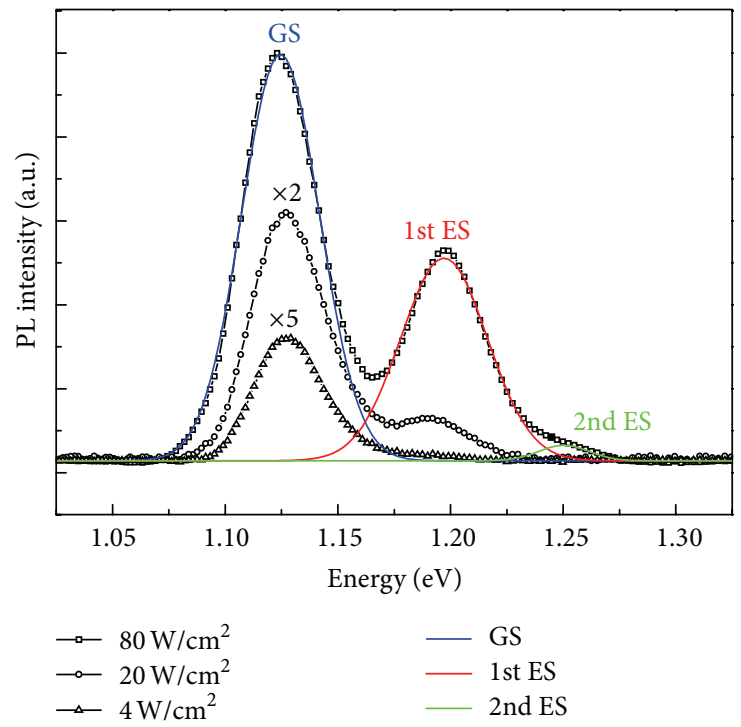

(a)
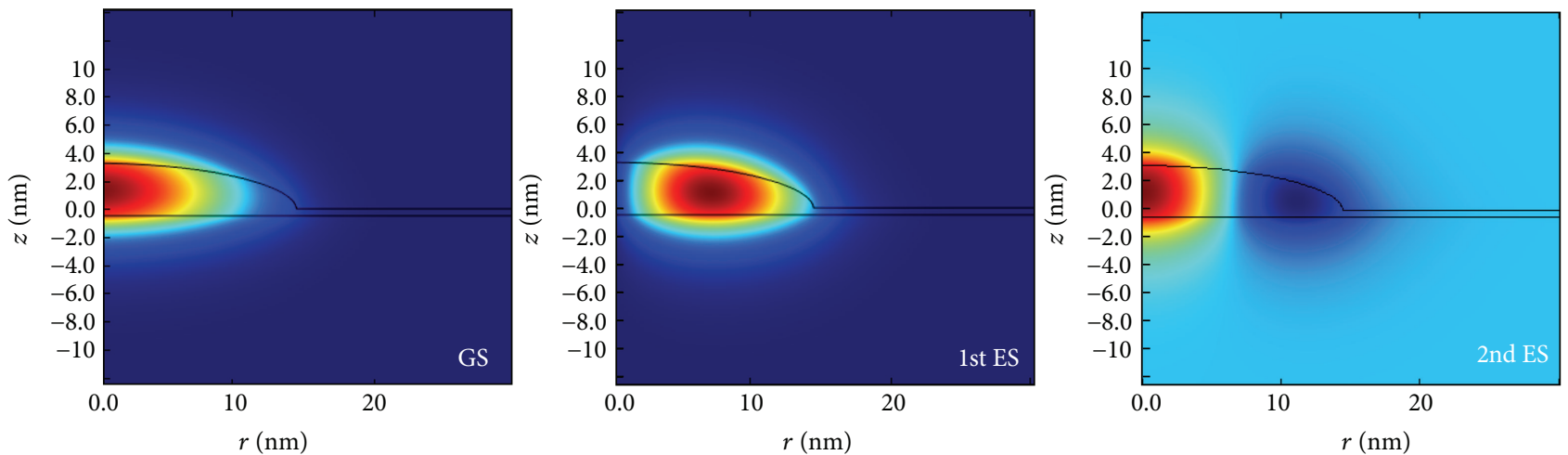

(b)

Figure 2: (a) Typical $10 \mathrm{~K} \mathrm{PL}$ spectra recorded at various excitation density at $80 \mathrm{Wcm}^{-2}$ (squares), $20 \mathrm{Wcm}^{-2}$ (circles), and $4 \mathrm{Wcm}{ }^{-2}$ (triangles). These spectra show the state filling effects with increasing the excitation power. The highest excitation density spectra show three emission bands deconvoluted by three Gaussians, namely, the optical transition energies arising from GS, 1st ES, and 2nd ES. (b) Electron envelop function calculated using the simulated buried dots size for the GS, 1st ES, and 2nd ES.

two excited states are given by Figure 3. The PL emission bands are found to be red shifted with decreasing the InAs material's deposition rate testifying an increased dots size. Additionally, for the same laser excitation power density, the 1st ES and GS emission peaks' intensity ratio increases from 0.5 for a growth rate of $0.069 \mathrm{ML} / \mathrm{s}$ to up to 1.3 for QDs deposited at $0.013 \mathrm{ML} / \mathrm{s}$. The relative enhancement of the 1st ES emission peaks' intensity indicates an increased number of the excess carriers available to populate the higher energy levels prior to their radiative recombination. This signifies also that the number of required photo carriers to saturate the GS energy levels decreases with decreasing the QDs deposition rate. The observed behavior, consequently, gives evidence of a pronounced decrease of the QDs' density. Accordingly, for high growth rate, the QDs are constrained to be prematurely formed because of the limited atom surface diffusion length [5-7]. However, for lower growth rate, the number of incoming surface atoms get reduced which increases their diffusion length. It becomes possible for them to reach existing islands where they get incorporated instead of being constrained to form additional islands. This induces a reduction of the QDs density and an increase of their size in conformity with AFM observations. Indeed, morphological studies have shown a monotonic decrease of the QDs density with a pronounced height enhancement with decreasing the growth rate $[6,7]$.

Knowing that the same InAs nominal material thickness has been deposited for all samples, the observed PL behavior is well explained by the decrease of the QDs density in favor of the formation of bigger dots' size. However, quantitative information on the realistic size evolution of the buried dots' size is missing considering the changes that may occur to the covered dots upon the deposition of the capping layer material $[23,24]$.

By considering a lens shaped QD with $1 \mathrm{ML}$ thick pure InAs WL, as stated in the theoretical consideration part, we 
TABLE 1: Experimental and calculated optical transition energies for different growth rates. Also presented are the simulated QDs height, base diameter, and corresponding aspect ratio.

\begin{tabular}{lccccccrrr}
\hline \multirow{2}{*}{ Growth rate $(\mathrm{ML} / \mathrm{s})$} & \multicolumn{2}{c}{$\mathrm{GS}(\mathrm{eV})$} & \multicolumn{2}{c}{ 1st ES $(\mathrm{eV})$} & \multicolumn{2}{c}{ 2nd ES (eV) } & \multirow{2}{*}{$h(\mathrm{~nm})$} & \multirow{2}{*}{$D(\mathrm{~nm})$} & \multirow{2}{*}{ AR } \\
& Exp. & Calc. & Exp. & Calc. & Exp. & Calc. & & \\
\hline 0.013 & 1.054 & 1.057 & 1.124 & 1.122 & 1.19 & 1.196 & 5.5 & 30 \\
0.026 & 1.070 & 1.069 & 1.139 & 1.14 & 1.204 & 1.206 & 4.6 & 30 \\
0.043 & 1.090 & 1.091 & 1.168 & 1.164 & 1.244 & 1.246 & 4.1 & 0.153 \\
0.069 & 1.124 & 1.126 & 1.197 & 1.193 & 1.26 & 1.264 & 3.3 & 28 & 0.146 \\
\hline
\end{tabular}

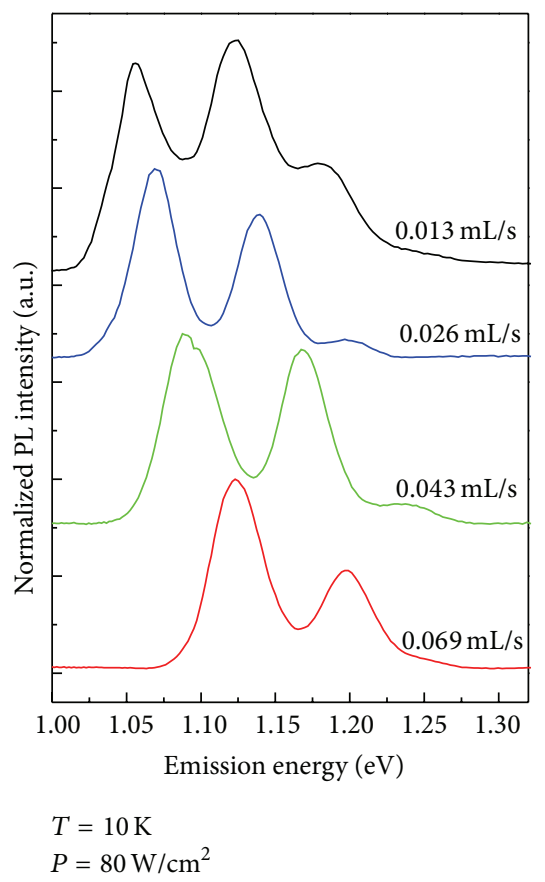

Figure 3: $10 \mathrm{~K}$ PL spectra obtained from InAs/GaAs QD samples obtained at $10 \mathrm{~K}$ with an excitation density of $80 \mathrm{~W} / \mathrm{cm}^{2}$ for InAs deposition rate $0.069 \mathrm{ML} / \mathrm{s}$ (red line), $0.043 \mathrm{ML} / \mathrm{s}$ (green line), $0.026 \mathrm{ML} / \mathrm{s}$ (blue line), and $0.013 \mathrm{ML} / \mathrm{s}$ (black line).

have tuned QD aspect ratio to fit the experimental optical transition energies. The results are shown in Figure 4, where the emission energies arising from the GS, 1st ES, and 2nd ES measured as the relative maximum of the corresponding PL emission peak are plotted with matched calculated values as a function of the deposition rate.

The good agreement within some $\mathrm{meV}$ allows gaining realistic size estimation for buried QDs. The results of calculated optical transition energies are presented in Table 1 together with the experimental values and simulated optimum buried QDs height, diameter, and AR.

For more quantitative analysis, the simulated QDs height and aspect ratio as well as the average uncovered dots height measured by AFM [7] have been plotted in Figure 5 as a function of the growth rate. The results show also a monotonic decrease of the dots AR with increasing the growth rate. The proposed method succeeds in giving the same asymptotic evolution of the QDs height with much smaller values. The observed large height difference between buried and

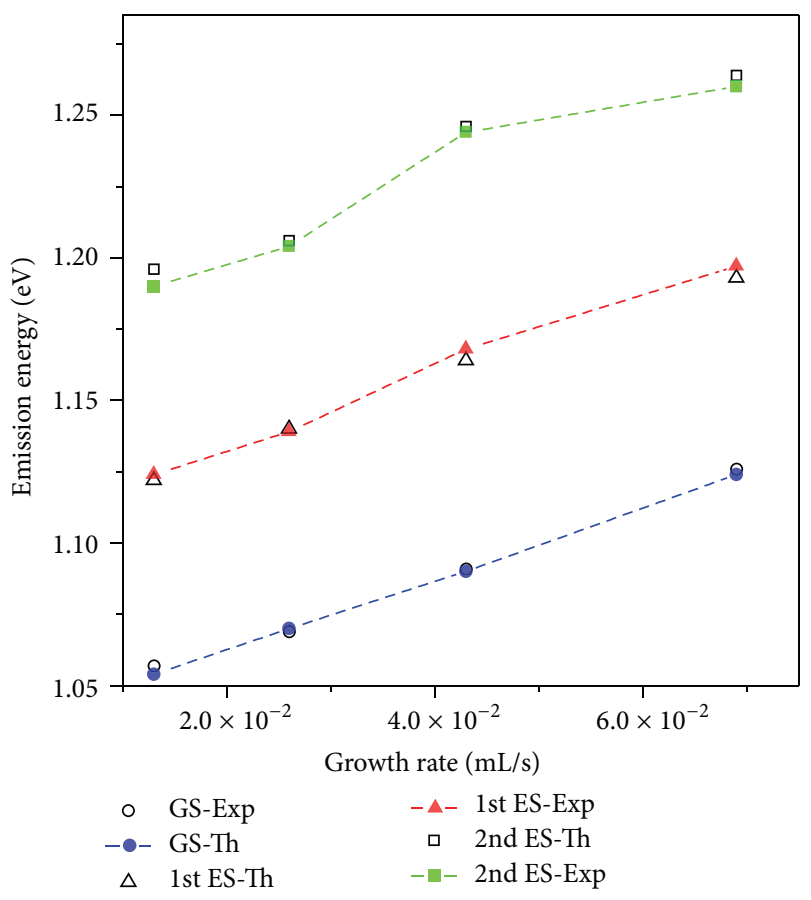

FIGURE 4: Calculated and experimental PL emission energies from the GS (circles), 1st ES (triangles), and 2nd ES (squares) as a function of the growth rate. The empty symbols refer to the calculated optical transition energies and the filled symbols to the experimental values.

uncapped QDs can have several cumulative reasons. Indeed, the AFM tip artifact can lead to an overestimation of the uncovered QDs size [25]. Additionally, the capping process has been shown to induce a shrinkage of the QDs size [26]. It is also worth mentioning that neglecting the intermixing effects in the present model might provide smaller QDs size [22].

Considering the relative variation of the QDs height and base diameter, the QDs height seems to be the most affected parameter by the growth rate variation within the adopted range in this work.

\section{Conclusion}

The correlation between high excitation density PL measurements and numerical solving of single particle one band Schrödinger equation in the frame of the effective mass approximation allowed estimating a realistic buried QDs 


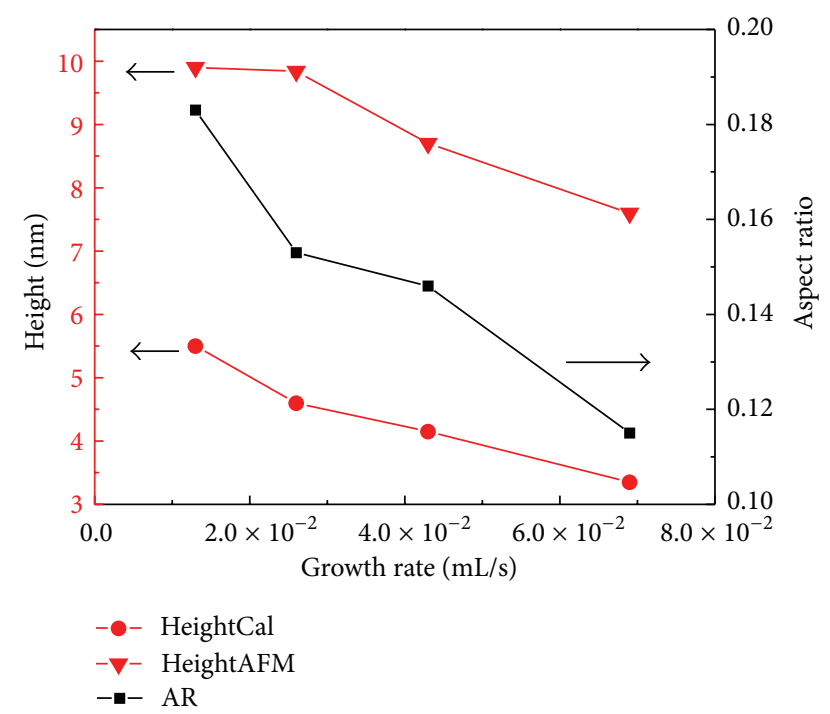

FIGURE 5: Simulated height and aspect ratio dependence on the growth rate. Also shown is the AFM driven average dots height from uncapped QDs.

size with application to truck the evolution of the covered InAs/GaAs QDs size and aspect ratio dependence on the growth rate. We found that the enhancement of the QDs size induced by the lowering of the growth rate is mainly due to the increase of the average dots height.

The proposed method is however general and can be applied to any QDs system if provided the ability to measure the optical transition energies issued from the ground and excited states.

\section{Conflict of Interests}

The authors declare that there is no conflict of interests regarding the publication of this paper.

\section{Acknowledgment}

The first author would like to extend his sincere appreciation to the Deanship of Scientific Research at King Saud University for funding this work through Research Group no. RG-1436014.

\section{References}

[1] H. Liu, T. Wang, Q. Jiang et al., "Long-wavelength InAs/GaAs quantum-dot laser diode monolithically grown on Ge substrate," Nature Photonics, vol. 5, pp. 416-419, 2011.

[2] I. J. Luxmoore, R. Toro, O. Del Pozo-Zamudio et al., "III-V quantum light source and cavity-QED on silicon," Scientific Reports, vol. 3, article 1239, 2013.

[3] S. Chen, M. Tang, Q. Jiang et al., "InAs/GaAs quantum-dot superluminescent light-emitting diode monolithically grown on a Si substrate," ACS Photonics, vol. 1, no. 7, pp. 638-642, 2014.

[4] L. Chu, M. Arzberger, G. Böhm, and G. Abstreiter, "Influence of growth conditions on the photoluminescence of self-assembled
InAs/GaAs quantum dots," Journal of Applied Physics, vol. 85, no. 4, pp. 2355-2362, 1999.

[5] P. B. Joyce, T. J. Krzyzewski, G. R. Bell, T. S. Jones, E. C. Le Ru, and R. Murray, "Optimizing the growth of $1.3 \mu \mathrm{m}$ InAs/GaAs quantum dots," Physical Review B, vol. 64, no. 23, Article ID 235317, 2001.

[6] M. J. da Silva, A. A. Quivy, S. Martini, T. E. Lamas, E. C. F. da Silva, and J. R. Leite, "Large InAs/GaAs quantum dots with an optical response in the long-wavelength region," Journal of Crystal Growth, vol. 278, no. 1-4, pp. 103-107, 2005.

[7] B. Ilahi, L. Sfaxi, E. Tranvouez et al., "Toward long wavelength low density InAs/GaAs quantum dots," Physics Letters A, vol. 357, no. 4-5, pp. 360-363, 2006.

[8] E. C. Le Ru, P. Howe, T. S. Jones, and R. Murray, "Strainengineered InAs/GaAs quantum dots for long-wavelength emission," Physical Review B, vol. 67, Article ID 165303, 2003.

[9] Z. D. Fang, Z. Gong, Z. H. Miao et al., "Effect of the InAlAs and InGaAs combination strain-reducing layer on $1.3 \mu \mathrm{m}$ emission self-assembled InAs/GaAs quantum dots," Journal of Physics D: Applied Physics, vol. 37, no. 7, pp. 1012-1016, 2004.

[10] O. Nasr, M. H. H. Alouane, H. Maaref, F. Hassen, L. Sfaxi, and B. Ilahi, "Comprehensive investigation of optical and electronic properties of tunable InAs QDs optically active at O-band telecommunication window with (In)GaAs surrounding material," Journal of Luminescence, vol. 148, pp. 243-248, 2014.

[11] M. Henini, "Properties and applications of quantum dot heterostructures grown by molecular beam epitaxy," Nanoscale Research Letters, vol. 1, no. 1, pp. 32-45, 2006.

[12] P. B. Joyce, T. J. Krzyzewski, G. R. Bell et al., "Effect of growth rate on the size, composition, and optical properties of InAs/ GaAs quantum dots grown by molecular-beam epitaxy," Physical Review B, vol. 62, no. 16, pp. 10891-10895, 2000.

[13] J. X. Chen, A. Markus, A. Fiore et al., "Tuning InAs/GaAs quantum dot properties under Stranski-Krastanov growth mode for $1.3 \mu \mathrm{m}$ applications," Journal of Applied Physics, vol. 91, p. 6710, 2002.

[14] B. Ilahi, L. Sfaxi, H. Maaref, G. Bremond, and G. Guillot, "Long wavelength vertically stacked InAs/GaAs(001) quantum dots with a bimodal size distribution: optical properties and electronic coupling," Superlattices and Microstructures, vol. 36, no. 1-3, pp. 55-61, 2004.

[15] G. Katsaros, A. Rastelli, M. Stoffel et al., "Evolution of buried semiconductor nanostructures and origin of stepped surface mounds during capping," Applied Physics Letters, vol. 89, no. 25, Article ID 253105, 2006.

[16] A. J. Williamson, L. W. Wang, and A. Zunger, “Theoretical interpretation of the experimental electronic structure of lensshaped self-assembled InAs/GaAs quantum dots," Physical Review B, vol. 62, no. 19, pp. 12963-12977, 2000.

[17] C. Pryor, "Geometry and material parameter dependence of InAs/GaAs quantum dot electronic structure," Physical Review $B$, vol. 60, no. 4, pp. 2869-2874, 1999.

[18] I. Vurgaftman and J. R. Meyer, "Band parameters for III-V compound semiconductors and their alloys," Journal of Applied Physics, vol. 89, no. 11, p. 5815, 2001.

[19] S. Lee, O. L. Lazarenkova, P. von Allmen, F. Oyafuso, and G. Klimeck, "Effect of wetting layers on the strain and electronic structure of InAs self-assembled quantum dots," Physical Review B-Condensed Matter and Materials Physics, vol. 70, no. 12, Article ID 125307, 2004.

[20] http://www.comsol.com/. 
[21] A. D. B. Maia, E. C. F. da Silva, A. A. Quivy, V. Bindilatti, V. M. de Aquino, and I. F. L. Dias, "Simulation of the electronic properties of $\mathrm{In}_{x} \mathrm{Ga}_{1-x}$ As quantum dots and their wetting layer under the influence of indium segregation," Journal of Applied Physics, vol. 114, Article ID 083708, 2013.

[22] M. Souaf, M. Baira, O. Nasr et al., "Investigation of the InAs/ GaAs quantum dots' size: dependence on the strain reducing layer's position," Materials, vol. 8, no. 8, pp. 4699-4709, 2015.

[23] G. Costantini, A. Rastelli, C. Manzano et al., "Interplay between thermodynamics and kinetics in the capping of InAs/GaAs(001) quantum dots," Physical Review Letters, vol. 96, Article ID 226106, 2006.

[24] F. Ferdos, S. Wang, Y. Wei, A. Larsson, M. Sadeghi, and Q. Zhao, "Influence of a thin GaAs cap layer on structural and optical properties of InAs quantum dots," Applied Physics Letters, vol. 81, no. 7, pp. 1195-1197, 2002.

[25] K.-I. Shiramine, S. Muto, T. Shibayama et al., "Tip artifact in atomic force microscopy observations of InAs quantum dots grown in Stranski-Krastanow mode," Journal of Applied Physics, vol. 101, no. 3, Article ID 033527, 2007.

[26] K. Yamaguchi, Y. Saito, and R. Ohtsubo, "Size-shrinkage effect of InAs quantum dots during a GaAs capping growth," Applied Surface Science, vol. 190, no. 1-4, pp. 212-217, 2002. 

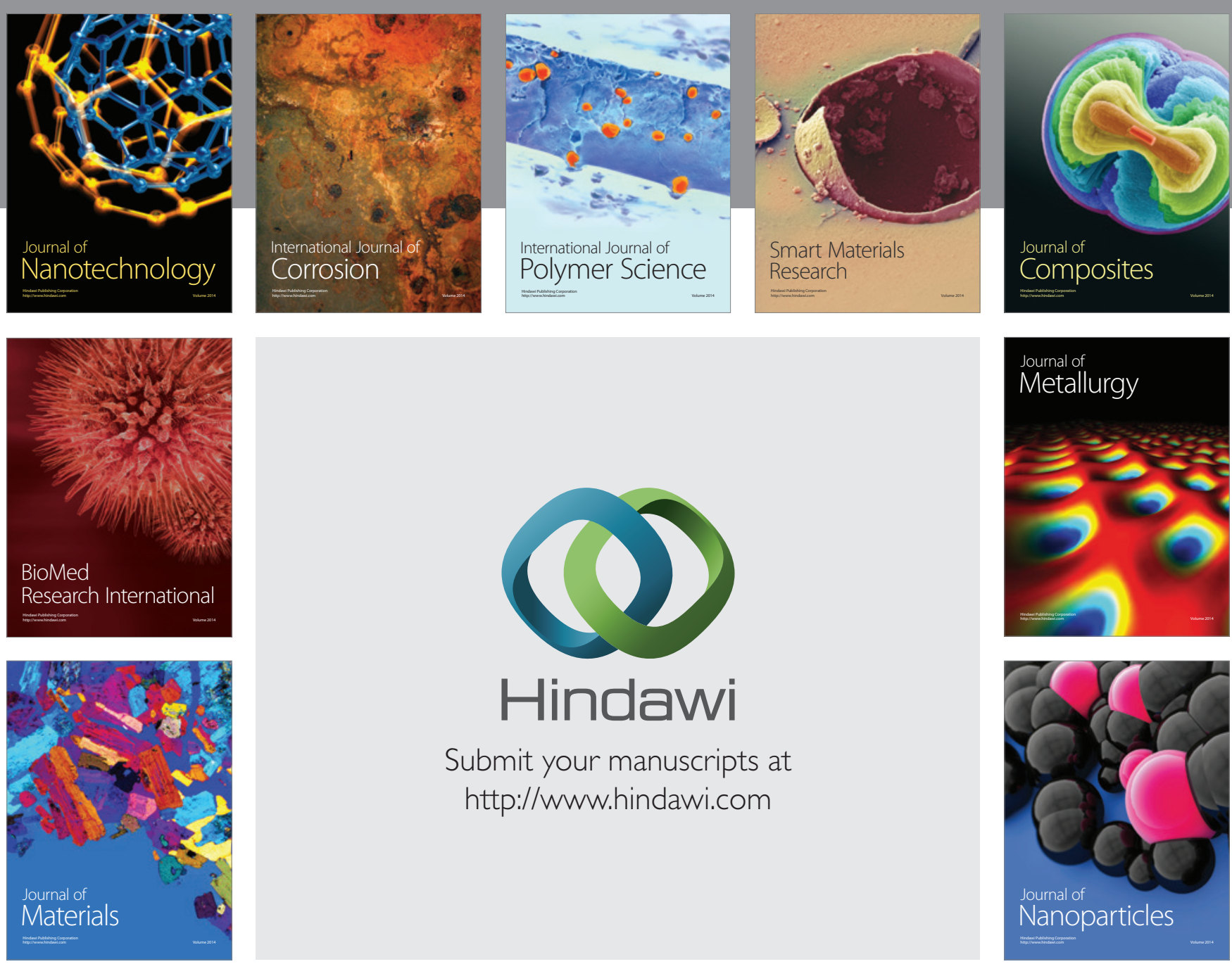

Submit your manuscripts at http://www.hindawi.com
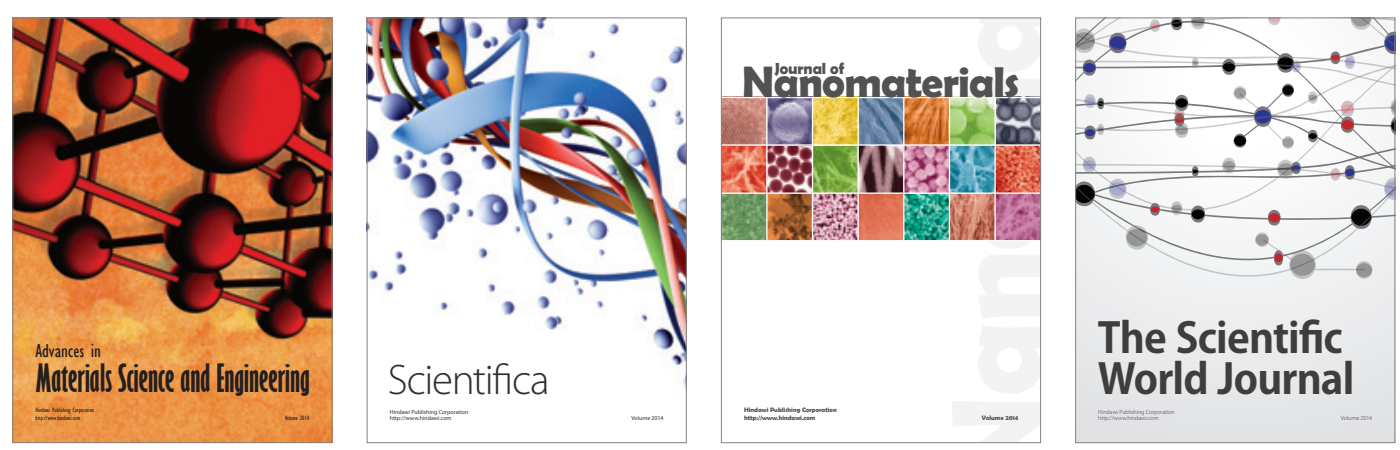

\section{The Scientific World Journal}
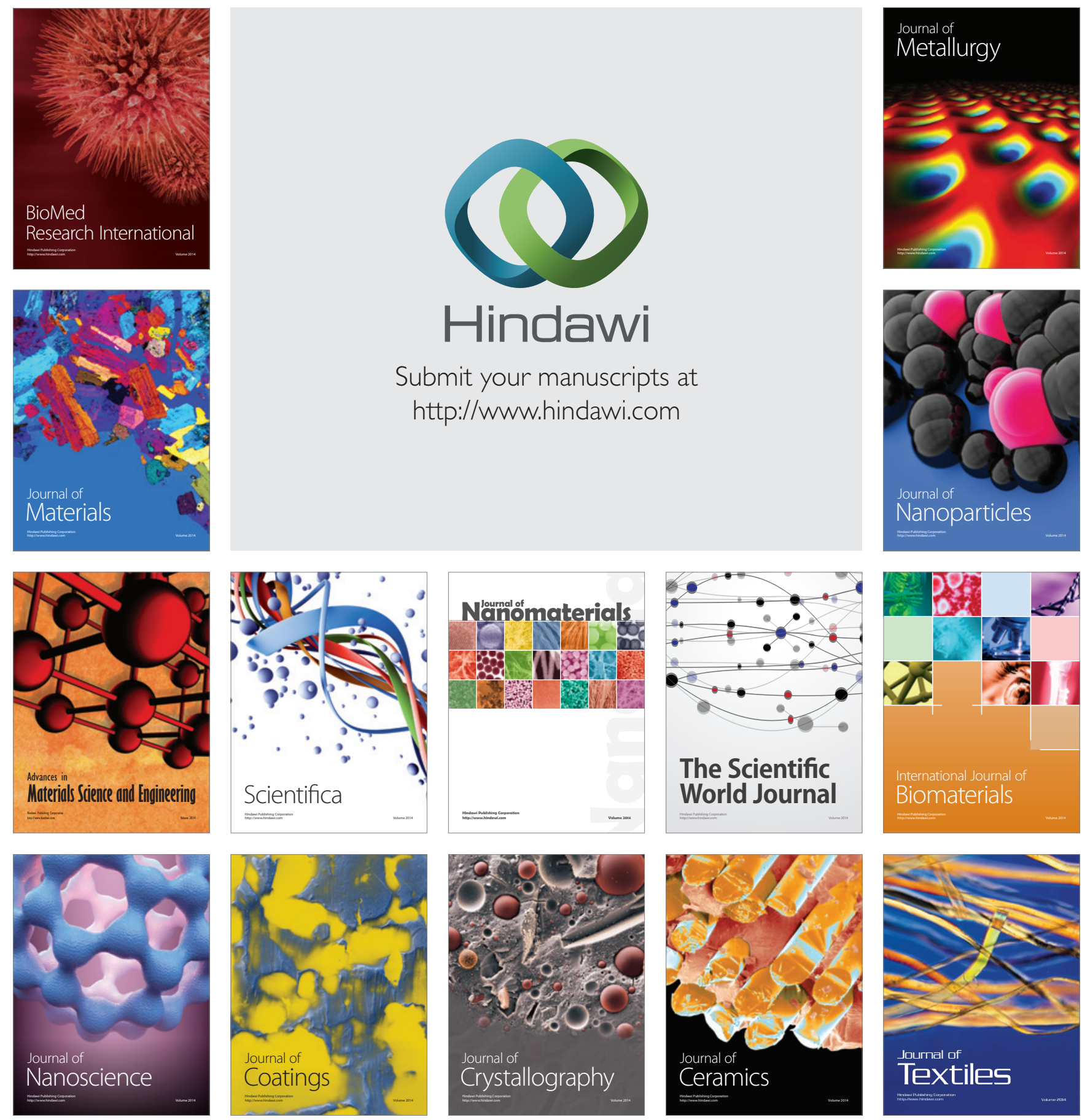